\title{
Effects of prenatal alcohol exposure on child behaviour outcomes at age five: Lifeways Cross-Generational Cohort Study
}

\author{
E. Mullen ${ }^{1}$, C. Murrin ${ }^{1,2}$, J. Mehegan ${ }^{2}$ and C.C. Kelleher ${ }^{2}$ \\ ${ }^{1}$ UCD School of Agriculture \& Food Science, University College Dublin, Republic of Ireland and \\ ${ }^{2}$ School of Public Health, Physiotherapy \& Sport Science, University College Dublin, Republic of Ireland
}

The association between heavy Prenatal Alcohol Exposure (PAE) and adverse behavioural and cognitive outcomes in offspring is well established $^{(1)}$. The effects of low-moderate levels of PAE are currently unknown. Prior to 2007, the Department of Health guidance did not provide strict recommendations on alcohol avoidance during pregnancy. Previous analysis of pregnant mothers of the Lifeways Cohort, recruited between 2001 and 2003, showed that many women continued to drink alcohol during pregnancy ${ }^{(2)}$. The aim of this study was to identify whether different levels of PAE were associated with child behavioural outcomes at age five years.

The mothers $(n=1124)$ were recruited from two Irish maternity hospitals and completed a baseline self-reported questionnaire during their first trimester ${ }^{(3)}$. Type and estimated volume of alcohol consumption was self-reported using a food frequency questionnaire which reflected their food intake since becoming pregnant. Alcohol intake was classified according to the average number of units of alcohol consumed per week. Maternal socio-demographic variables were also self-reported in the questionnaire. Mothers and the proband child were followed up when the children were aged 5 years. Ethical approval was granted for each phase of data collection.

A 36 item child behaviour questionnaire was completed by each mother to determine child behavioural difficulties. Parents responded using a three point Likert scale. Ten behavioural domains were investigated including internalising and externalising behaviour problems, hyperactivity and aggression. Each domain was scored according to the standardised Strengths and Difficulties Questionnaire (SDQ) classification scoring tool ${ }^{(4)}$. The scores were totalled and reported as both a continuous variable (median score) and categorical variable. The SDQ Original Three-Band classification along with internal referencing was used to classify child behavioural domains as a categorical variable ${ }^{(4)}$. Categories were identified as either 'normal' or 'borderline/at risk'. Kruskal-Wallis tests and Chi-squared statistical tests were conducted to determine differences or associations between child behavioural outcomes and maternal alcohol intakes.

Of the $n=1106$ mothers who completed the FFQ at baseline, 52.4\% abstained from alcohol during pregnancy, $23.4 \%$ were occasional drinkers, $14 \%$ were low level drinkers and $9.8 \%$ were moderate-heavy level drinkers. Mothers of older age $(\geqslant 30$ years, $\mathrm{p}<0.001)$, higher education status $(\geqslant$ third level, $\mathrm{p}=0.002)$ and married/cohabiting $(\mathrm{p}=0.010)$ were significantly more likely to consume higher levels of alcohol. Of the $n=585$ mother-child dyads included at follow-up, approximately $20 \%$ of children were categorised into the 'borderline/at risk' group for each child behavioural domain. No significant differences or associations were observed in child behavioural difficulties across levels of PAE. Further multivariate analysis is required to adjust for potential factors that may confound the association.

1. Sampson PD, Streissguth AP, Bookstein FL, et al. (1997) Teratology 56, 317-326.

2. Murrin C, Fallon U, Hannon F, et al. (2007) IMJ 100, suppl 12-15.

3. O'Mahony D, Fallon U, Hannon F, et al. (2007) IMJ 100, suppl 3-6.

4. Goodman R (1997) J Child Psychol Psychiatry. 38, 581-586. 